\title{
CDUCT-LaRC Status - Shear Layer Refraction and Noise Radiation
}

\author{
Douglas M. Nark*and F. Farassat ${ }^{\dagger}$ \\ NASA Langley Research Center, Hampton, Virginia
}

\begin{abstract}
A proposed boundary condition accounting for shear layer effects within the Ffowcs Williams-Hawkings radiation module of the CDUCT-LaRC code is investigated. The development and numerical justification of the boundary condition formulation are reviewed. An initial assessment of the effectiveness of the shear layer correction is conducted through comparison with experimental data. Preliminary results indicate that the correction provides physically meaningful modifications of the baseline predicted directivity patterns. Trends of peak directivity steepening and shifting that appeared in predicted patterns were found to follow similar structures in measured data, particularly at higher radiation angles.
\end{abstract}

\section{Nomenclature}

$c \quad$ sound speed, $\mathrm{m} / \mathrm{s}$

$c_{e} \quad$ effective wave speed, $\mathrm{m} / \mathrm{s}$

$k, k_{x} \quad$ free space and axial wave numbers, $1 / \mathrm{m}$

$M_{1}, M_{2} \quad$ mean flow Mach number in regions 1 and 2

$M_{d} \quad$ Mach number in the duct

$m \quad$ circumferential mode number

$\vec{n} \quad$ unit normal to the shear layer

$R \quad$ annular duct radius $\left(r_{\text {outer }}-r_{\text {inner }}\right), \mathrm{m}$

$R_{S} \quad$ local shear layer radius, $\mathrm{m}$

$\vec{u} \quad$ fluid velocity, $\mathrm{m} / \mathrm{s}$

$\overrightarrow{V_{s}} \quad$ modal trace velocity on shear layer surface, $\mathrm{m} / \mathrm{s}$

$z_{1}, z_{2} \quad$ acoustic impedance on shear layer surface adjacent to regions 1 and 2

Symbols:

$\rho$

ambient density, $\mathrm{kg} / \mathrm{m}^{2}$

$\omega$

circular frequency, $\mathrm{rad} / \mathrm{s}$

\footnotetext{
${ }^{*}$ Research Scientist, Structural Acoustics Branch, Research \& Technology Directorate, AIAA Member

${ }^{\dagger}$ Senior Theoretical Acoustician, Aeroacoustics Branch, Research \& Technology Directorate, AIAA Associate Fellow
} 


\section{Introduction}

The capability to efficiently predict acoustic radiation from aircraft engines with acceptable accuracy is a valuable tool in the development and assessment of low-noise concepts. The fan noise propagation and radiation code CDUCT-LaRC ${ }^{1}$ includes an acoustic radiation module that is based on the Ffowcs Williams- Hawkings (FW-H) equation with penetrable data surfaces. ${ }^{2}$ In the current implementation, the source surface is taken to be the inlet or exhaust planes for inlet or aft-fan cases, respectively. In many situations, this provides excellent results. However, there are limitations inherent in these source surface selections. The first issue can arise in both inlet and aft-fan cases when large off-axis radiation angles are of interest. In these situations, the shielding and edge effects of the engine cowl are not properly taken into account. This results in predictions that are essentially symmetric about the source surface. The second issue arises in aft-radiation cases and is associated with the refraction and possible amplification of sound waves as they propagate through the shear layer. This work focuses on the investigation of a proposed approach to account for shear layer effects within the framework of the CDUCT-LaRC code. The following discussion is broken into two main components: description of the model for shear layer refraction and application toward verification of the model through comparison with experimental data.

\section{Shear Layer Model}

The shear layer between the bypass flow and external stream can refract the sound waves radiated to the farfield. Radiation results can be improved by including this effect, as well as the reflection of the sound in the bypass region from the solid surface external to the bypass duct surrounding the core flow (see figure 1). One way to achieve this is to extend the CDUCT-LaRC bypass duct propagation calculation, which is based on the parabolic approximation, beyond the exhaust plane and to apply a boundary condition (BC) similar to an acoustic liner BC on the shear layer. Although presented previously,, 3 a brief review of this boundary condition may be useful. The development is based on the satisfaction of two conditions on the shear layer:

1. The particle displacement must be continuous across the shear layer.

2. The acoustic pressure must be continuous across the shear layer.

The first result in the derivation involves the acoustic velocity normal to the shear layer. This follows a derivation of the liner BC by Myers ${ }^{4}$ as presented by Farassat and Dunn. ${ }^{5}$ The subscripts 0 and 1 are used for the background and acoustic quantities. The particle displacement amplitude normal to the shear layer is also denoted by $q_{3}$ and the time dependence is harmonic with the factor $e^{-i \omega t}$. Following the notation of Farassat and Dunn ${ }^{5}$ [eq. (3) with $\varepsilon=1$ ], one may write

$$
\begin{aligned}
\frac{\partial q_{3}}{\partial t}=-i \omega q_{3} & =\left[q_{3} \overrightarrow{n_{0}} \cdot\left(\overrightarrow{n_{0}} \cdot \nabla \overrightarrow{u_{0}}\right)+\overrightarrow{u_{1}} \cdot \overrightarrow{n_{0}}\right]^{1} \\
& =\left[q_{3} \overrightarrow{n_{0}} \cdot\left(\overrightarrow{n_{0}} \cdot \nabla \overrightarrow{u_{0}}\right)+\overrightarrow{u_{1}} \cdot \overrightarrow{n_{0}}\right]^{2}
\end{aligned}
$$

where the superscripts 1 and 2 designate regions 1 and 2 in figure $1, \vec{u}$ is the fluid velocity, and $\vec{n}$ is the unit normal to the shear layer.

From equation 11, one finds

$$
q_{3}=\frac{-u_{1 n}^{1}}{i \omega+\left[\overrightarrow{n_{0}} \cdot\left(\overrightarrow{n_{0}} \cdot \nabla \overrightarrow{u_{0}}\right)\right]^{1}}=\frac{-u_{1 n}^{2}}{i \omega+\left[\overrightarrow{n_{0}} \cdot\left(\overrightarrow{n_{0}} \cdot \nabla \overrightarrow{u_{0}}\right)\right]^{2}}
$$


and

$$
u_{1 n}^{2}=\frac{i \omega+\left[\overrightarrow{n_{0}} \cdot\left(\overrightarrow{n_{0}} \cdot \nabla \overrightarrow{u_{0}}\right)\right]^{2}}{i \omega+\left[\overrightarrow{n_{0}} \cdot\left(\overrightarrow{n_{0}} \cdot \nabla \overrightarrow{u_{0}}\right)\right]^{1}} u_{1 n}^{1} .
$$

This result shows that if $u_{1 n}^{1}$ is found on the shear layer from the parabolic approximation method in region 1 , then $u_{1 n}^{2}$ on the external side of the shear layer may be calculated.

Next, the derivation of the impedance, $z_{2}$, on the side of the shear layer adjacent to region 2 is presented beginning with the model and assumptions First, the shear layer is taken to be infinitely thin, an assumption previously utilized in equation 3 . Second, the barber pole pattern of the modes present in the flow region is assumed to persist in the flow region inside the shear layer. This barber pole pattern has a trace velocity on the shear layer surface in the direction normal to itself. Figure 2 shows this trace velocity, $\overrightarrow{V_{s}}$, and the method by which it is calculated. This figure also shows a local tangent plane to the shear layer surface which is cylindrical. Assuming the acoustic pressure in a mode to be proportional to $\exp \left[-i\left(\omega t-m \theta-k_{x} x\right)\right]$ where $m$ is the circumferential mode number, $x$ is the axial distance, and $k_{x}$ is the axial wave number, it follows that

$$
\begin{gathered}
\dot{\theta}=\frac{\omega}{m}, \dot{x}=\frac{\omega}{k_{x}} \\
R_{s} \dot{\theta}=\frac{\omega / c_{1}}{m} R_{s} c_{1}=\frac{k R_{s}}{m} c_{1} \\
\dot{x}=\frac{\omega / c_{1}}{k_{x}} c_{1}=\frac{k}{k_{x}} c_{1} \\
V_{s}=\left\|\vec{V}_{s}\right\|=k c_{1} \sqrt{\left(\frac{R_{s}}{m}\right)^{2}+\frac{1}{k_{x}^{2}}} .
\end{gathered}
$$

Here, $R_{s}$ is the local radius of the shear layer (assumed almost constant), $k=\omega / c_{1}$, and $c_{1}$ is the local speed of sound in region 1 of the shear layer.

From Morse and and Ingard ${ }^{6}$ (equation 11.1.21), the impedance, $z_{2}$, is

$$
z_{2}=\frac{\rho_{2} c_{2}}{\left(1+M_{2} \cos \phi\right) \sin \phi}
$$

where $M_{2}=u_{2} / c_{2}$ is the Mach number based on the background flow in region 2 and $\phi$ is the preferred direction of propagation of plane waves in region 2 . This angle is found purely by kinematic considerations. Figure 3 shows the geometry in the local plane containing $\vec{V}_{s}$ and the local normal to the shear layer which is along axis 2 . Let $\vec{e}=(\sin \phi, \cos \phi)$ be the direction normal to the wavefront. The effective wave speed in region $2, \overrightarrow{c_{e}}$, is

$$
\overrightarrow{c_{e}}=c_{2} \vec{e}+\overrightarrow{u_{2}} \cos \psi
$$

where $\cos \psi=\dot{x} / V_{s}$ is shown in figure 2. The projection of $\overrightarrow{c_{e}}$ on the shear layer is equal to the trace speed $V_{s}$. This gives

$$
V_{s}=u_{2} \cos \psi+c_{2} \sin \phi
$$

${ }^{a}$ Note that until the end of this section, we work with the zeroth order quantities (background flow, time independent quantities). For simplicity of notation, we denote regions 1 and 2 by subscripts. 
from which it is seen that

$$
\sin \phi=\frac{V_{s}-u_{2} \cos \psi}{c_{2}}
$$

Using this in equation 8 leads to

$$
z_{2}=\frac{\rho_{2} c_{2}\left(V_{s}-u_{2}\right)}{V_{s} \sqrt{\left(V_{s}-u_{2}\right)^{2}-c_{2}^{2}}} .
$$

The final step in the formulation is that

$$
z_{1}=\frac{u_{1 n}^{2}}{u_{1 n}^{1}} z_{2}
$$

and equation 3 provides an expression for $u_{1 n}^{2} / u_{2 n}^{1}$.

\section{Implementation}

Implementation of the impedance boundary condition rests on the calculation of two main quantities. First, the ratio of particle velocity external to the shear layer (region 2) to the particle velocity internal to the shear layer (region 1), $u_{1 n}^{2} / u_{2 n}^{1}$ must be known (see eq. 3). Determination of this ratio requires knowledge of the background flow velocity vectors and the unit normal to the shear layer surface. The background flow velocity vectors and location of the shear layer are available from the mean flow solution supplied for the propagation calculations. The NonUniform Rational B-Splines (NURBS) techniques utilized within the grid generation and redistribution functionality of CDUCT-LaRC provide the ability to define a general shear layer surface and therefore, the unit normal to the shear layer.

Secondly, the impedance on the external side of the shear layer, $z_{2}$, must also be specified. As seen in equation 11, calculation of $z_{2}$ requires knowledge of the local radius of the shear layer, $R_{s}$, the circumferential mode number, $m$, and the axial wave number, $k_{x}$. The possible issues involved in obtaining these quantities in general has been discussed previously ${ }^{3}$ For the cases considered in this work, the possible complications are avoided by using the value of $z_{2}$ obtained for the lowest radial order of the highest order circumferential mode.

With the required quantities in hand, the shear layer model may incorporated into the propagation module of CDUCT-LaRC. The shear layer surface defines the outer surface of a 'pseudo-duct' that extends from the exhaust plane of the bypass duct. An inner 'pseudo-duct' surface must also be defined, which in a typical engine application is at least partially defined by the outer surface of the core cowl. The source plane of the 'pseudo-duct' is defined by the exit surface of the final block used in the internal propagation calculation. The acoustic potential values on this exit plane are also used directly to specify the generic source and an additional propagation calculation is subsequently performed within the 'pseudo-duct'. The exposed surfaces of the 'pseudo-duct' are then used as FW-H source surfaces in the radiation calculations. In order to illustrate the implementation further, and provide some verification of the approach, some sample calculations are presented. 


\section{Application}

The verification cases considered are the by-product of previous calculations performed in conjunction with colleagues at NASA Glenn Research Center. In this previous work, CDUCT-LaRC predictions were made for the NASA Glenn Active Noise Control Fan (ANCF) rig and compared with results obtained using the Eversman finite element code. ${ }^{7}$ The ANCF rig is a low speed fan testbed designed for noise reduction concept testing and code verification/development. ${ }^{8}$ In addition to collection of farfield data, the ANCF allows for the insertion of a rotating microphone rake ${ }^{9}$ to measure the interaction modes of the ducted fan. Therefore, for selected test configurations, induct rotating rake measurements and farfield acoustics measurements may be obtained. A general schematic of the axi-symmetric rig is presented in figure 4.

Although not a focus of this work, some points may be made concerning the preliminary comparisons between CDUCT-LaRC and the Eversman finite element code. First, as a result of initial problem formulation, the capability to directly input rotating rake data was added to CDUCTLaRC. Secondly, to illustrate the effects of including the proposed shear layer correction, comparison of the CDUCT-LaRC and Eversman inlet and aft predictions for the $(2,0)$ mode at one BPF $(533 \mathrm{~Hz})$ are provided in figure 5. Aft baseline and shear layer corrected CDUCT-LaRC predictions are included. Note that for these aft results, inclusion of the shear layer correction increases the peak directivity level and shifts its location forward. Overall, the initial comparisons were encouraging and provided motivation to pursue further investigation of this approach to accounting for shear layer effects. Having introduced the ANCF configuration and some of the initial effort, comparisons with measured data for aft radiation are discussed.

A range of source frequencies (1, 2, and 3 BPF) and flow speeds (1700 through 2000 RPM) were considered. The corresponding rotating rake data for each case was used to specify the modal sources. The expected Tyler-Sofrin modes (based on 16 blades and 14 stators), with amplitude and phase for 1, 2, and $3 \mathrm{BPF}$ at each fan speed, are listed in tables 1 through 4 of the Appendix. Note that the source information corresponding to the various BPF values are separated within the tables and the duct Mach number, $M_{d}$, is supplied as well. The rotating rake data is supplied at an axial location of $x=61.6 \mathrm{in}$. The proximity to the exhaust plane ( $x=64.6 \mathrm{in}$.) leads to the use of a short duct section for the internal propagation calculations. Subsequently, to utilize the shear layer boundary condition, an external 'pseudo-duct' must be attached to the internal duct segment. Propagation calculations are then performed within the 'pseudo-duct' and the solution on the exposed surfaces used as the source surface for the FW-H radiation calculations. The farfield directivity obtained in this manner may depend on the axial extent of the 'pseudo-duct'. Therefore, the calculations were made for several 'pseudo-duct' lengths defined in terms of multiples of the annular duct radius, $R$, at the exhaust plane. Specifically, lengths equivalent to 1.0, 2.5, 5.0, and 10.0 multiples of the duct radius were investigated. As an example of the computational meshes used, the internal and 'pseudo-duct' meshes for the $2.5 R$ case are shown in figure 6 and represented with white and green wireframe, respectively. Note that, although the geometry is axi-symmetric, the calculations are performed over the fully three-dimensional geometry for verification purposes.

In this figure, it can also be seen that the shear layer is assumed to form a straight cylindrical surface. As such, the Mach number in the duct (internal and pseudo) is taken to be uniform and the external Mach number is set to zero. The aforementioned flow and source specifications were used to perform internal and 'pseudo-duct' propagation calculations. For the internal propagation calculation, the grid size was 21X89X9 in the radial, circumferential, and axial directions, respectively. All 'pseudo-ducts' used the same radial and circumferential dimension as the internal block. The 
axial grid sizes and typical wall-clock times for complete propagation and radiation predictions for each of the cases are provided in table 5. In this table, the EP case identifier refers to the baseline radiation calculation using the exhaust plane of the bypass duct as the FW-H source surface. Subsequent cases involving the shear layer boundary condition are referred to as SL:XX where XX refers to the length of the 'pseudo-duct' in multiples of the annular duct radius. Farfield data for the ANCF is available at 15 microphone locations evenly spaced along a 15 foot arc centered on the exhaust plane. Figure 7 shows the placement of the observer arc relative to the ANCF geometry.

As mentioned previously, predictions were performed for the first three blade passage frequencies for corrected fan speeds of 1700 to 2000 RPM. The results for each case are presented in figures 8 through 19 . Note that, for convenience, the remaining figures are placed at the end of the document. Looking first at the $1 \mathrm{BPF}$ results, it can be seen (figures 8, 11, 14, and 17) that the effect of the shear layer boundary condition is to increase and narrow the peak directivity, as well as shift it forward. This trend compares favorably with the measured data and the predictions match quite well at 2000 RPM. The more complex directivity patterns of the 2 BPF cases $9,12,15$, and 18) result in greater disparity between the predicted and measured farfield values. However, the shear layer correction tends to modify the fairly smooth baseline result and provide reasonable detection of peaks that begin to appear. In particular, the results at 1700 and 1800 RPM (figures 9, 12) show peaks in the modified directivity patterns that are similar to the measured data, particularly at the higher radiation angles. Finally, the comparisons between predicted and measured data at $3 \mathrm{BPF}$ (figures 10, 13, 16, and 19) are similar to those for the 2 BPF cases. The shear layer correction produces directivity patterns that are more directional relative to the baseline prediction. Additionally, the locations of the peaks appear to follow trends in the measured data, particularly at the higher radiation angles. Generally, the results are encouraging in that the shear layer correction produces modifications to the baseline predictions that more closely follow trends in the measured data. In several instances, the predicted directivity patterns show main peaks that are very much in line with the measured data in both angle and amplitude. Some of the discrepancies at forward radiation angles may be due to diffraction at the trailing edge of the nacelle. In the 2 BPF, 2000 RPM case however, some of the difficulties may be partially related to the source description. As seen in table 4 , the rotating rake data included information for the $m=4$ an -8 circumferential modes. However, from a Tyler-Sofrin analysis, a circumferential mode order of -10 is possible due to interaction at 2 BPF. At 2000 RPM, the source frequency is such that the $(-10,1)$ mode is just above cut-off. To gain some qualitative understanding of the effects of including this mode, a sample calculation was performed for the 2 BPF, 2000 RPM case in which the $(-10,1)$ mode was added to the original source. The amplitude of the mode was set to $2.0 \mathrm{~N} / \mathrm{m}^{2}$ and the phase was set to zero. For illustrative purposes, the predicted pattern was offset by $6 \mathrm{~dB}$ and also plotted (SLA: $10 \mathrm{R})$. From comparison of figures 18 and 20 , it can be seen that in this case that the inclusion of the $(-10,1)$ mode greatly improves the comparison with measured data. For the lower fan speeds however, the $(-10,1)$ is cut-off and the initial comment concerning trailing edge effects may be appropriate. Continued comparison with other codes, such as the Eversman finite element code, will provide further information on this issue. Additionally, further understanding may be gained by comparison with results presented by Mani ${ }^{10}$ and Munt. 11 


\section{Concluding Remarks}

In this work, the motivation for, and description of, a proposed boundary condition for the FW$\mathrm{H}$ acoustic radiation module of CDUCT-LaRC have been presented. Previous CDUCT-LaRC predictions for the ANCF rig have compared favorably with results obtained by colleagues at NASA Glenn using the Eversman finite element code. This led to further study of the effectiveness of a shear layer correction developed for inclusion in the FW-H radiation module of CDUCT- LaRC. Preliminary hardwall ANCF predictions were generated, using rotating rake data as source input, and compared with measured farfield data. Results indicate that the correction provides physically meaningful modifications of the baseline predicted directivity patterns. Some discrepancies at forward radiation angles were improved by inclusion of additional source modes, providing further illustration of the known importance of source specification. Some of the other discrepancies at forward radiation angles may be the results of edge effects encountered at the trailing edge of the nacelle. Further comparison with results from the Eversman finite element code, as well as work by Mani and Munt, will provide further insight into this matter. Overall, predicted trends of peak directivity steepening and shifting were found to follow similar structures in measured data, particularly at higher radiation angles.

\section{Acknowledgments}

The authors would like to thank Dan Sutliff (NASA Glenn Research Center) for assistance in obtaining the ANCF rotating rake and farfield data and Ed Envia (NASA Glenn Research Center) for generation of the Eversman finite element predictions included in this work.

\section{References}

\footnotetext{
${ }^{1}$ Nark, D. M., Farassat, F., Pope, D. S., and Vatsa, V., "The Development of the Ducted Fan Noise Propagation and Radiation Code CDUCT-LaRC," AIAA Paper 2003-3242, 2003.

${ }^{2}$ Brentner, K. B. and Farassat, F., "Analytical Comparison of the Acoustic Analogy and Kirchhoff Formulation for Moving Surfaces,” AIAA Journal, Vol. 36, No. 8, 1998, pp. 1379-1386.

${ }^{3}$ Nark, D. M., Farassat, F., Pope, D. S., and Vatsa, V., "A Model for Shear Layer Effects on Engine Noise Radiation," AIAA Paper 2004-2992, 2004.

${ }^{4}$ Myers, M. K., "On the Acoustic Boundary Condition in the Presence of Flow," Journal of Sound and Vibration, Vol. 71, No. 3, 1980, pp. 429-434.

${ }^{5}$ Farassat, F. and Dunn, M. H., "A Simple Derivation of the Acoustic Boundary Condition in the Presence of Flow," Journal of Sound and Vibration, Vol. 224, No. 2, 1999, pp. 384-386.

${ }^{6}$ Morse, P. M. and Ingard, K. U., Theoretical Acoustics, McGraw-Hill Book Company, 1968.

${ }^{7}$ Eversman, W., "Mapped Infinite Wave Envelope Elements for Acoustic Radiation in a Uniformly Moving Medium," Journal of Sound and Vibration, Vol. 224, 1999, pp. 665-687.

${ }^{8}$ Sutliff, D. L., Heidelberg, L. J., Elliott, D. M., and Nallasamy, M., "Baseline acoustic levels of the NASA active noise control fan rig," AIAA Paper 96-1745, 1996.

${ }^{9}$ Sutliff, D. L., "Rotating Rake Turbofan Duct Mode Measurement System,” NASA CR 213828, 2005.

${ }^{10}$ Mani, R., "Sound Propagation in Parallel Sheared Flows in Ducts: the Mode Estimation Problem," Proceedings of the Royal Society of London A, Vol. 371, 1980, pp. 393-412.

${ }^{11}$ Munt, R. M., "Acoustic Transmission Properties of a Jet Pipe with Subsonic Jet Flow: I. The Cold Jet Reflection Coefficient," Journal of Sound and Vibration, Vol. 142, No. 3, 1990, pp. 413-436.
} 


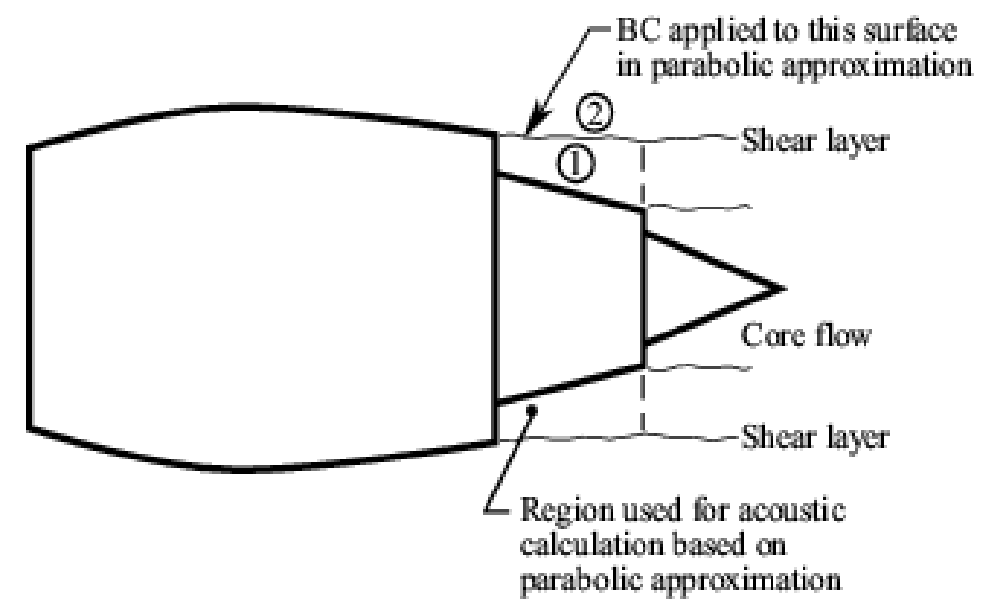

Figure 1. Boundary Regions for the Aft-Fan Geometry

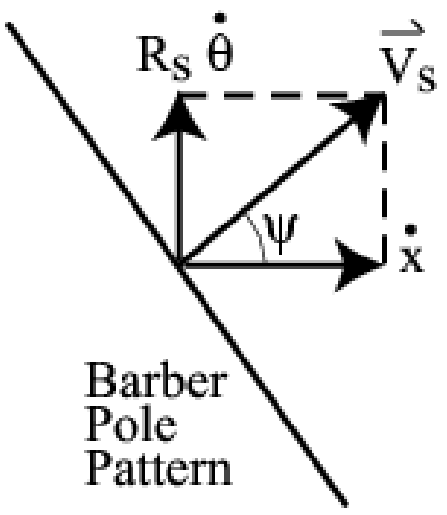

Figure 2. Trace Velocity Description

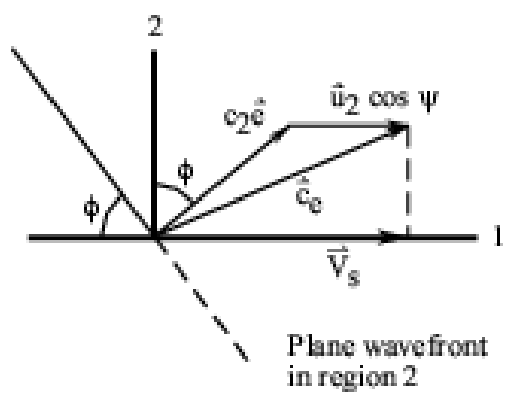

Figure 3. Effective Wave Speed Description

$$
8 \text { of } 19
$$




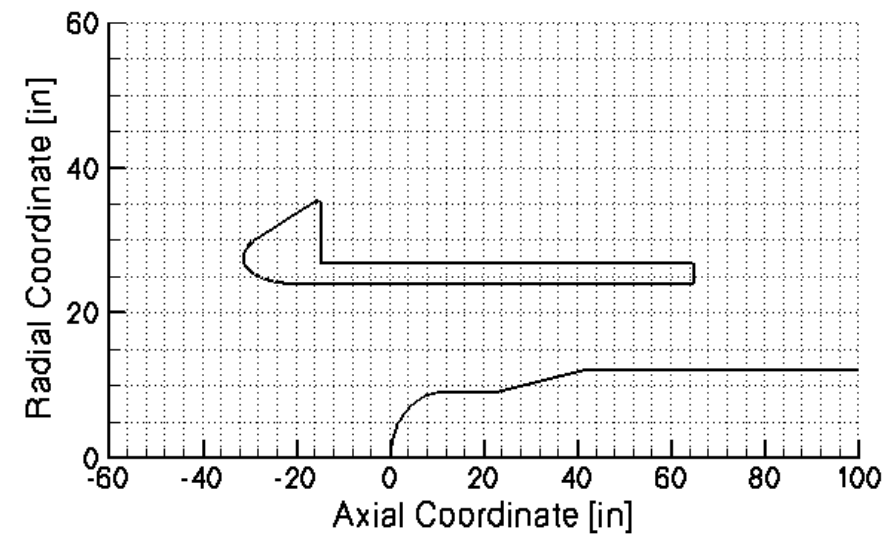

Figure 4. Schematic of NASA Glenn ANCF rig

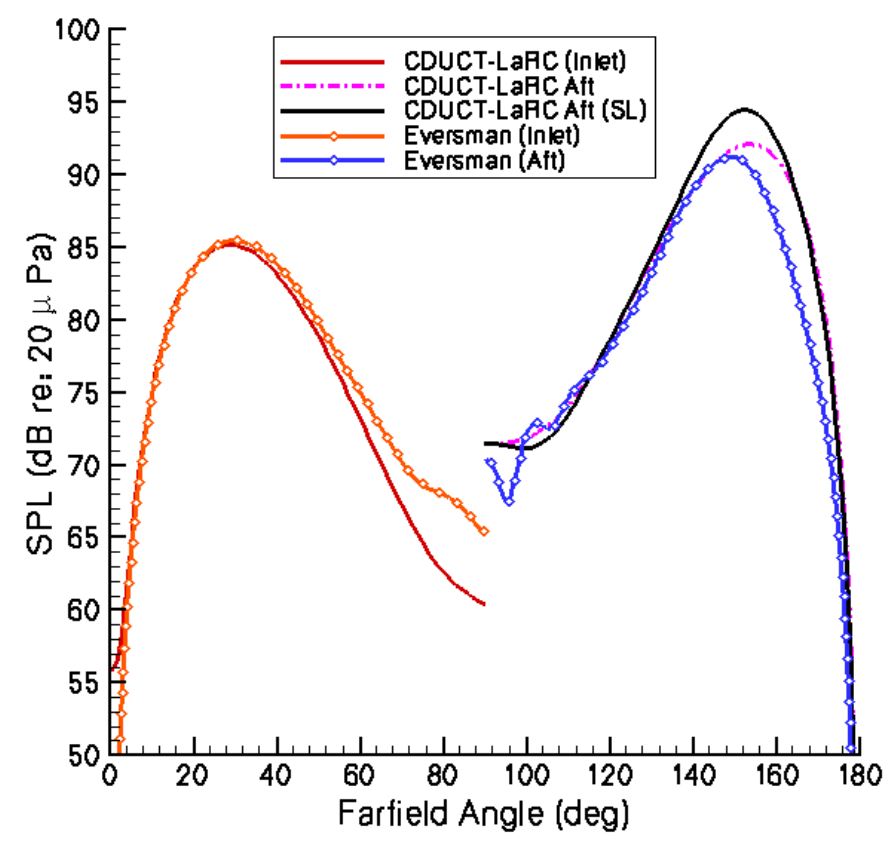

Figure 5. Initial Comparison of CDUCT-LaRC and Eversman Predictions [BPF $=533 \mathrm{~Hz},(2,0)$ mode]

$$
9 \text { of } 19
$$




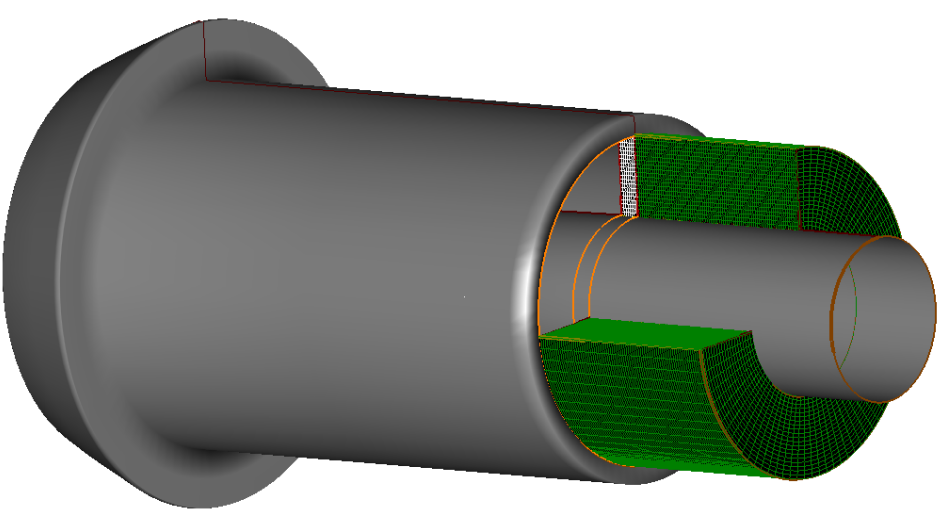

Figure 6. Internal and 'Pseudo-Duct' Computational Meshes (2.5R)

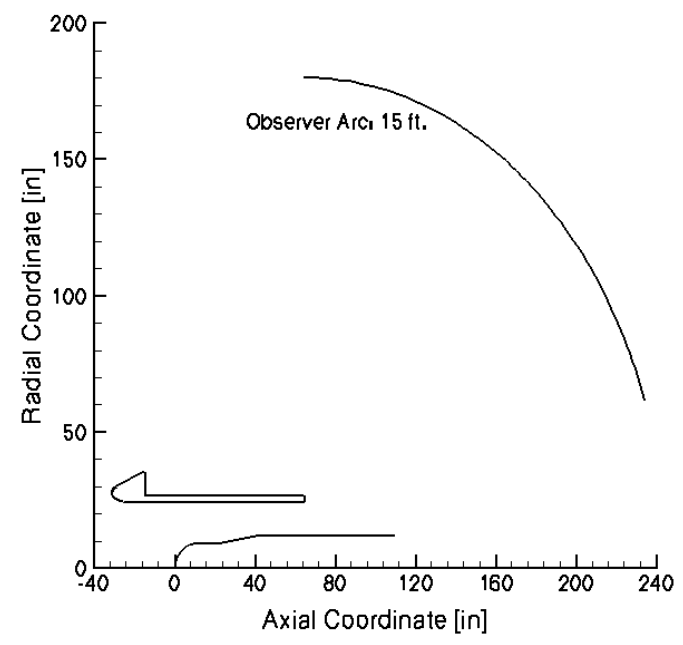

Figure 7. Observer Arc Location Relative to the ANCF geometry

\section{0 of 19}




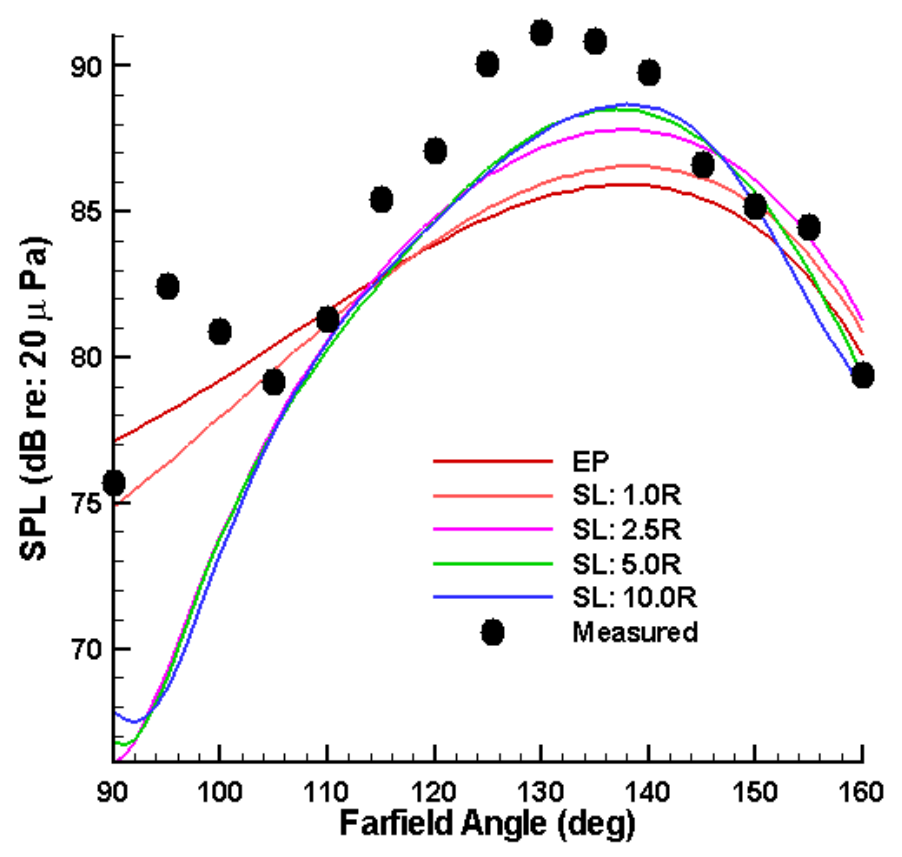

Figure 8. Aft ANCF prediction: 1700 RPM, $1 B P F(453 \mathrm{~Hz})$

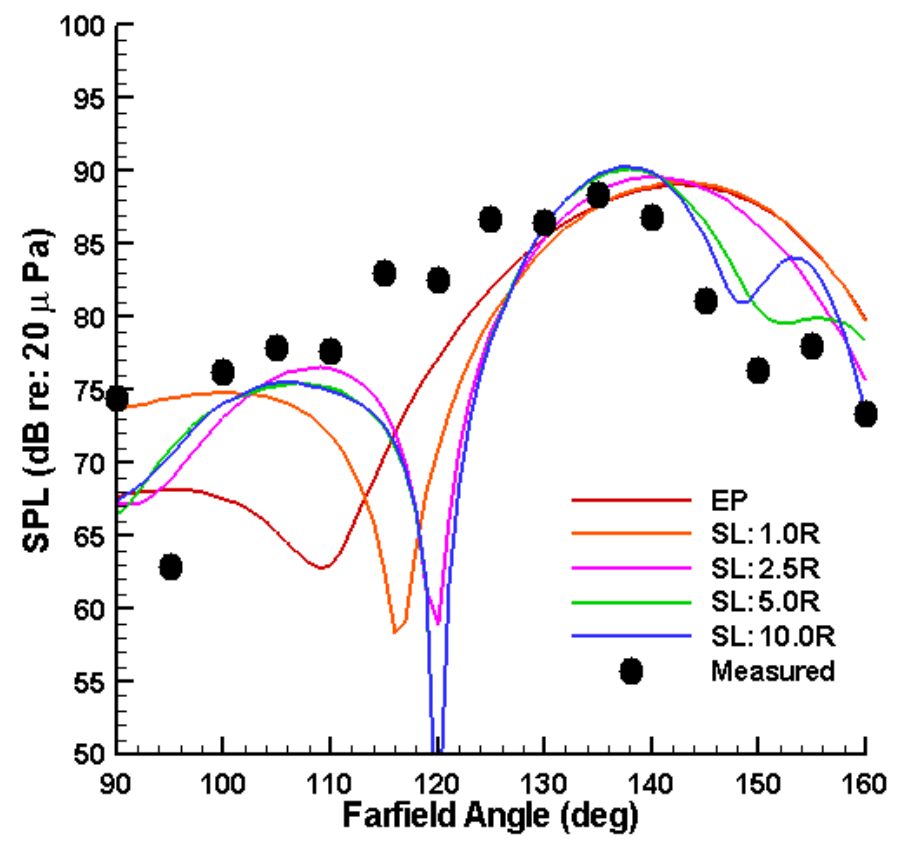

Figure 9. Aft ANCF prediction: 1700 RPM, 2BPF $(906 \mathrm{~Hz})$ 


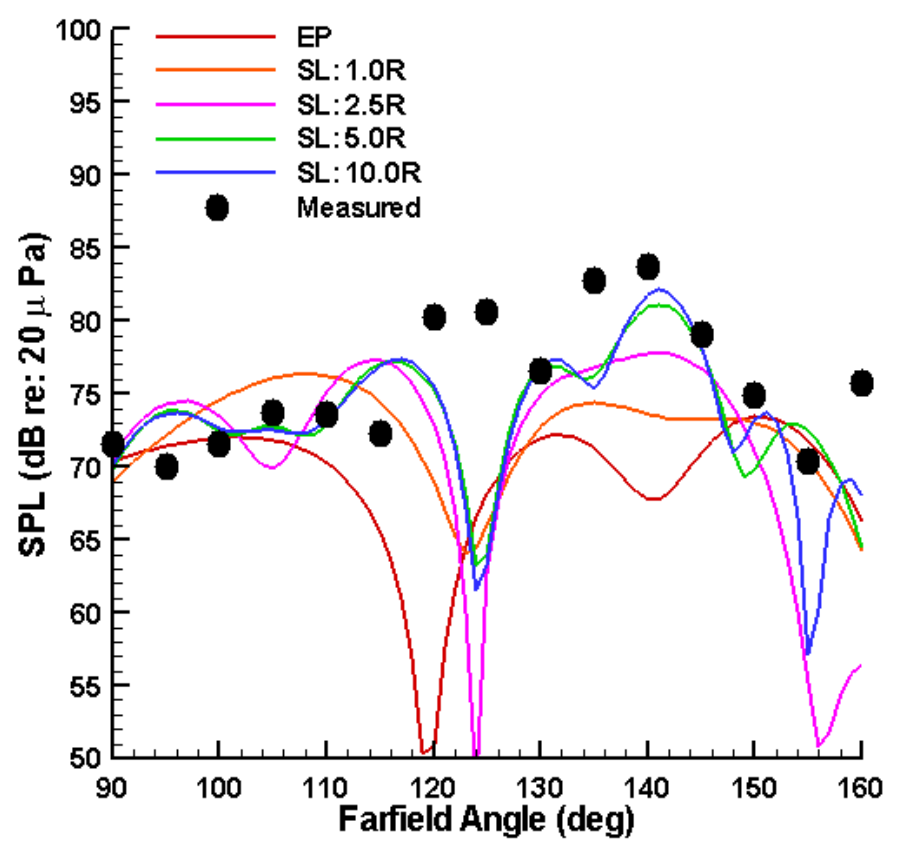

Figure 10. Aft ANCF prediction: 1700 RPM, 3BPF $(1360 \mathrm{~Hz})$

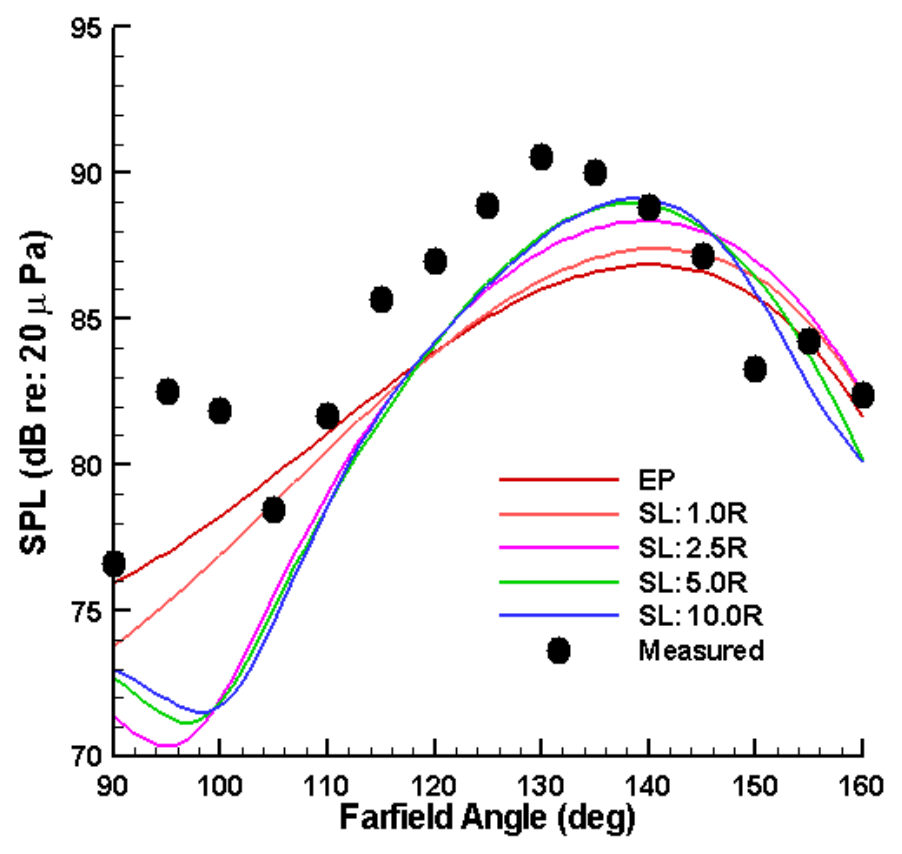

Figure 11. Aft ANCF prediction: $1800 \mathrm{RPM}, 1 \mathrm{BPF}(480 \mathrm{~Hz})$

\section{2 of 19}




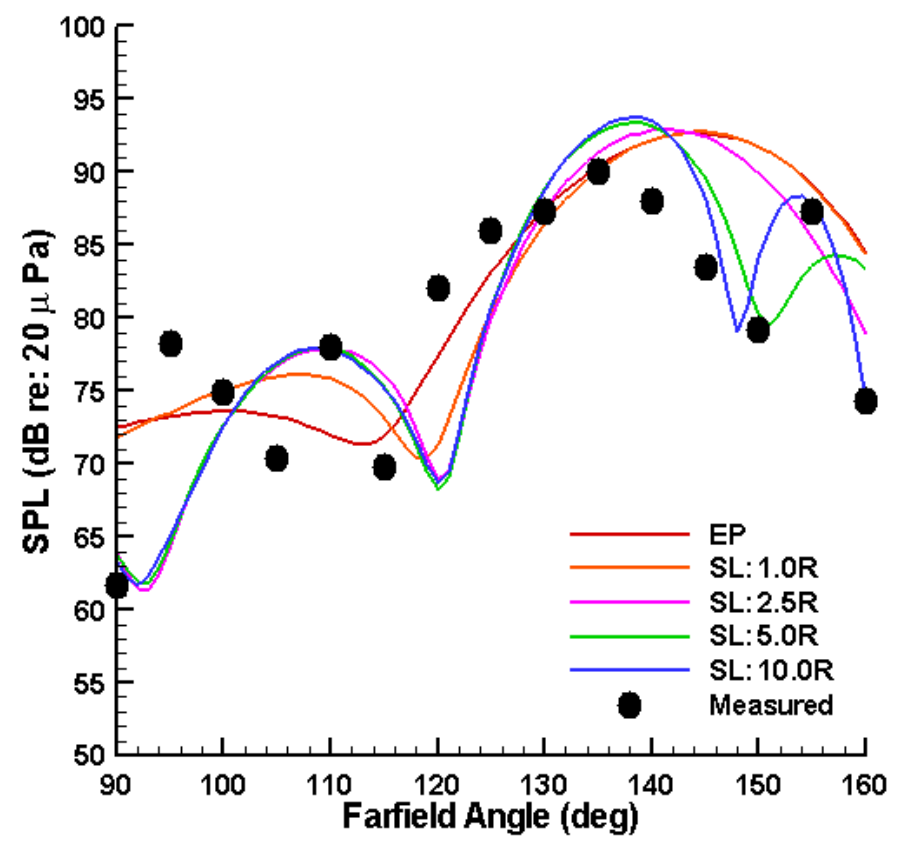

Figure 12. Aft ANCF prediction: 1800 RPM, 2BPF $(960 \mathrm{~Hz})$

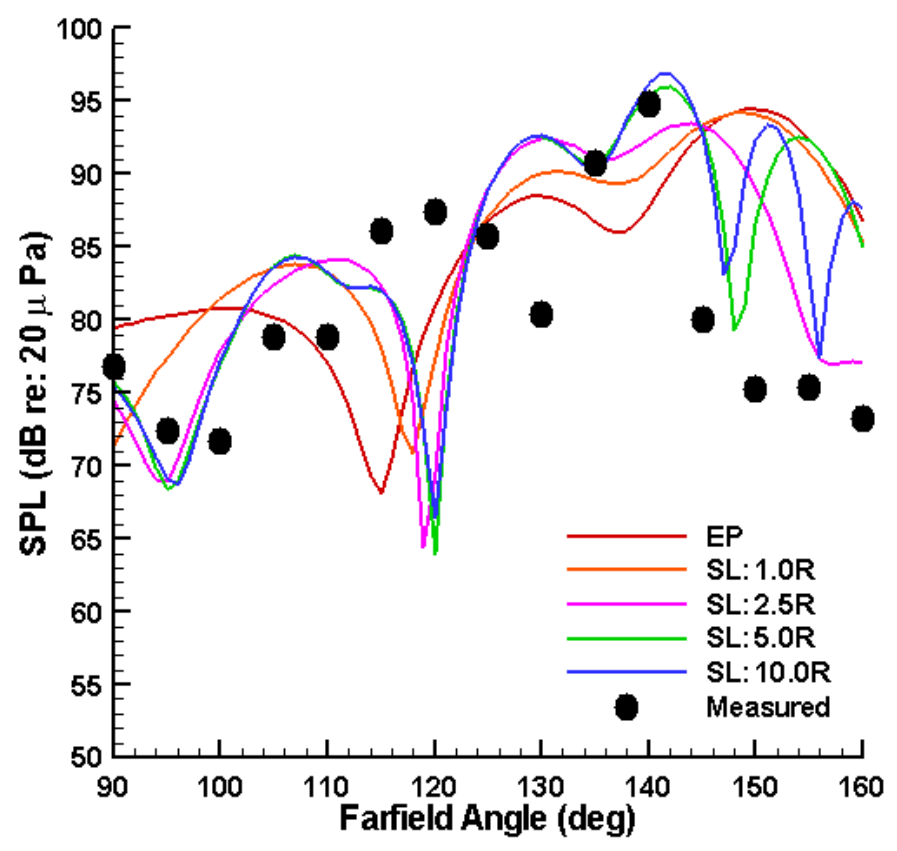

Figure 13. Aft ANCF prediction: $1800 \mathrm{RPM}, 3 \mathrm{BPF}(1440 \mathrm{~Hz})$

\section{3 of 19}




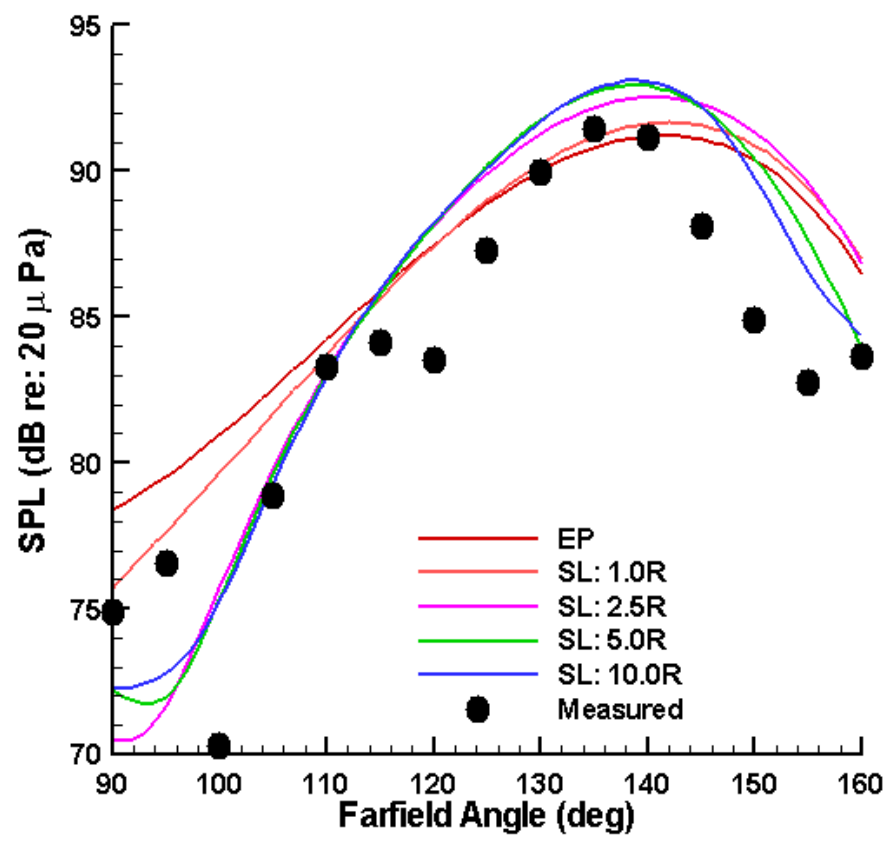

Figure 14. Aft ANCF prediction: 1900 RPM, 1BPF (507Hz)

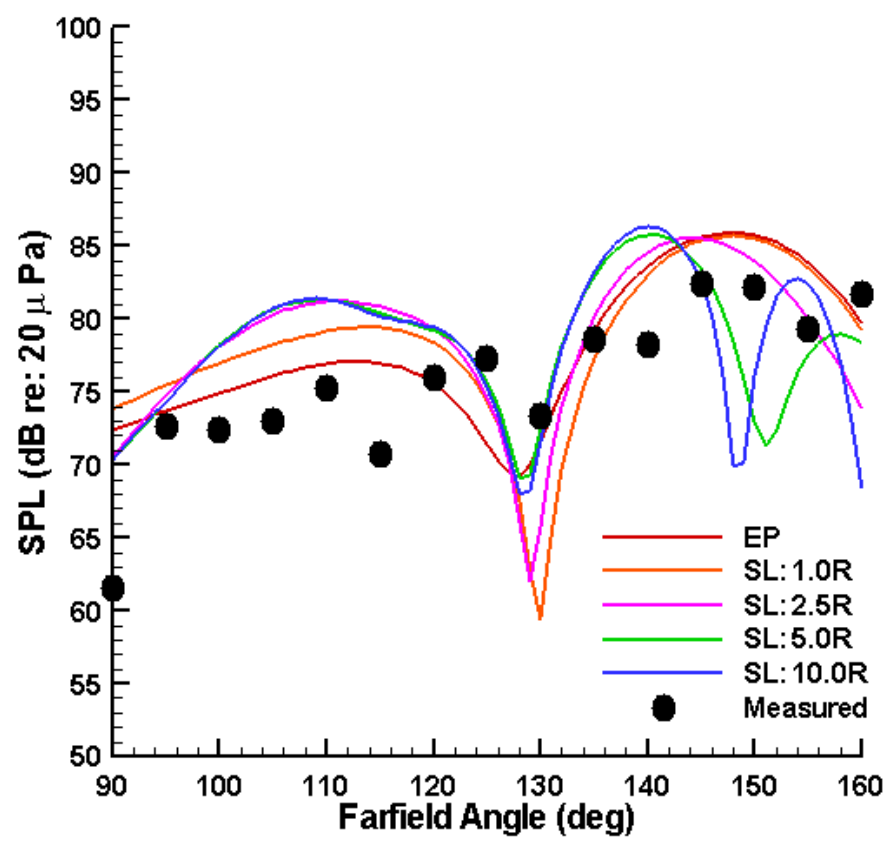

Figure 15. Aft ANCF prediction: 1900 RPM, 2BPF (1013 Hz)

\section{4 of 19}




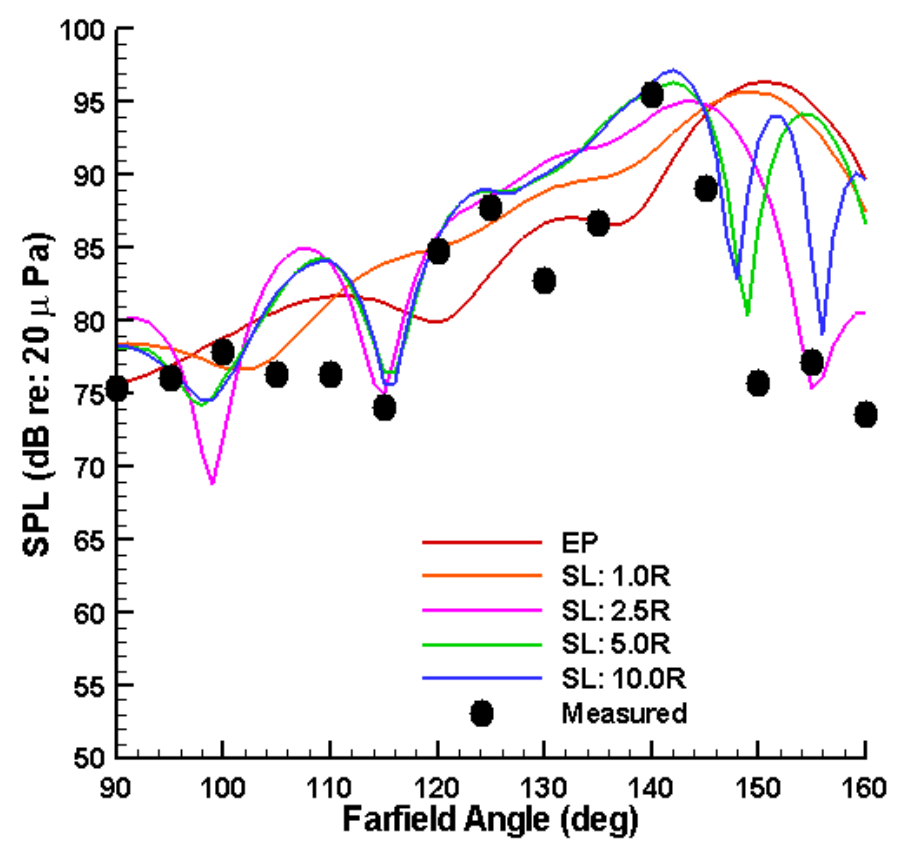

Figure 16. Aft ANCF prediction: 1900 RPM, 3BPF $(1520 \mathrm{~Hz})$

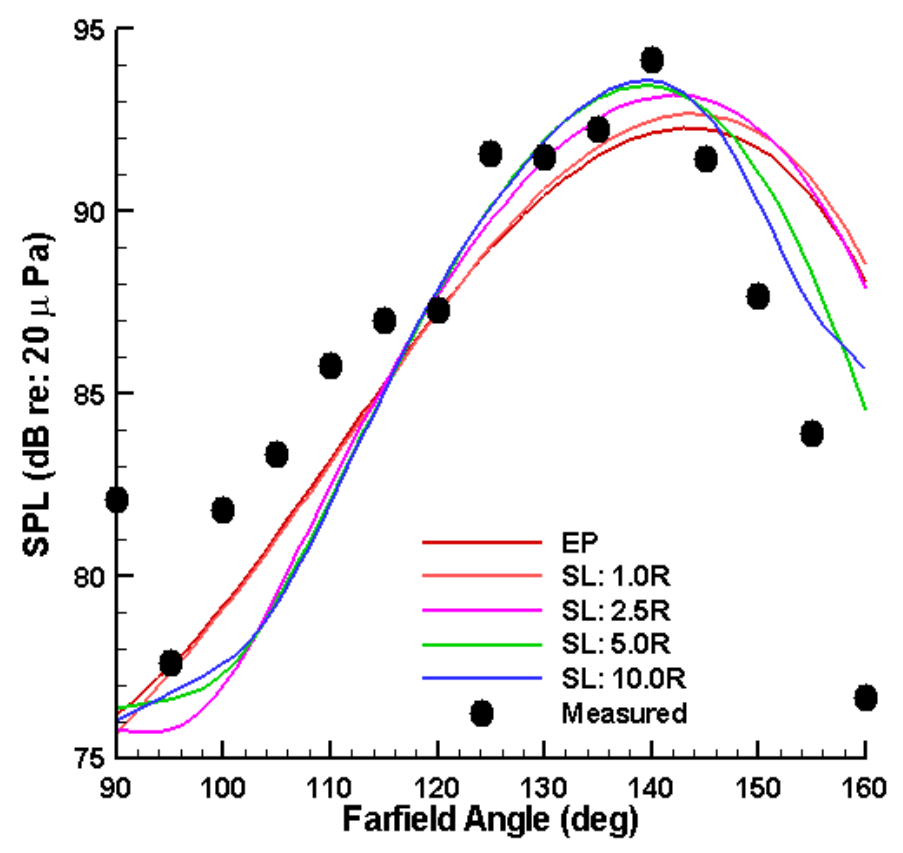

Figure 17. Aft ANCF prediction: $2000 \mathrm{RPM}, 1 \mathrm{BPF}(533 \mathrm{~Hz})$

\section{5 of 19}




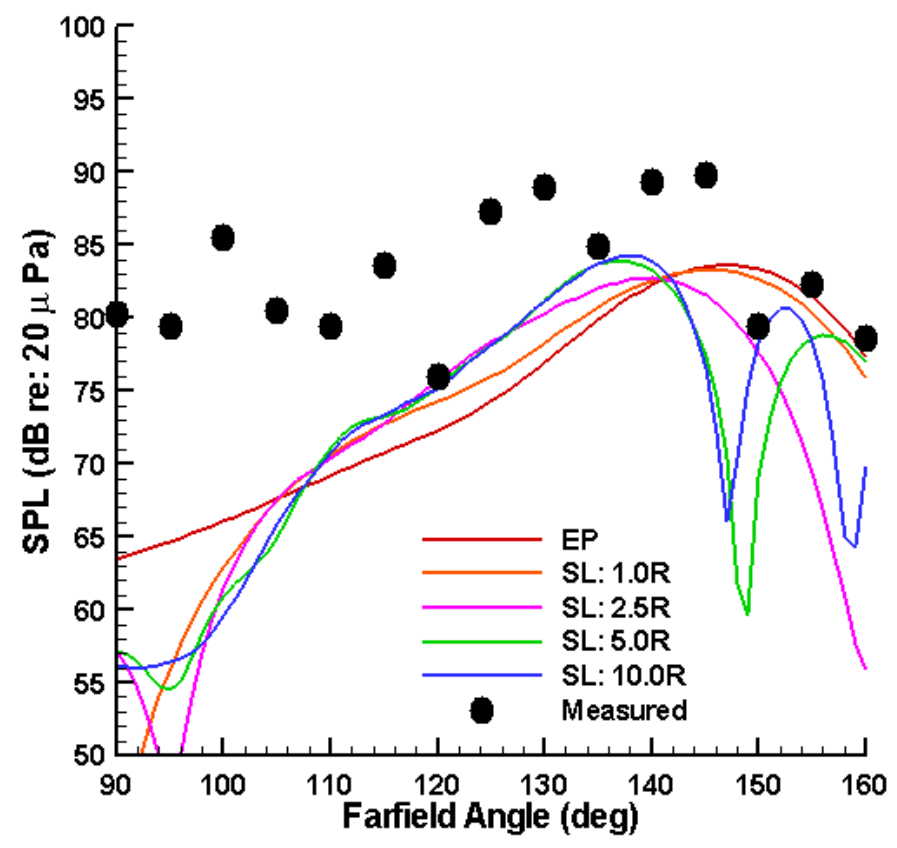

Figure 18. Aft ANCF prediction: 2000 RPM, 2BPF $(1066 \mathrm{~Hz})$

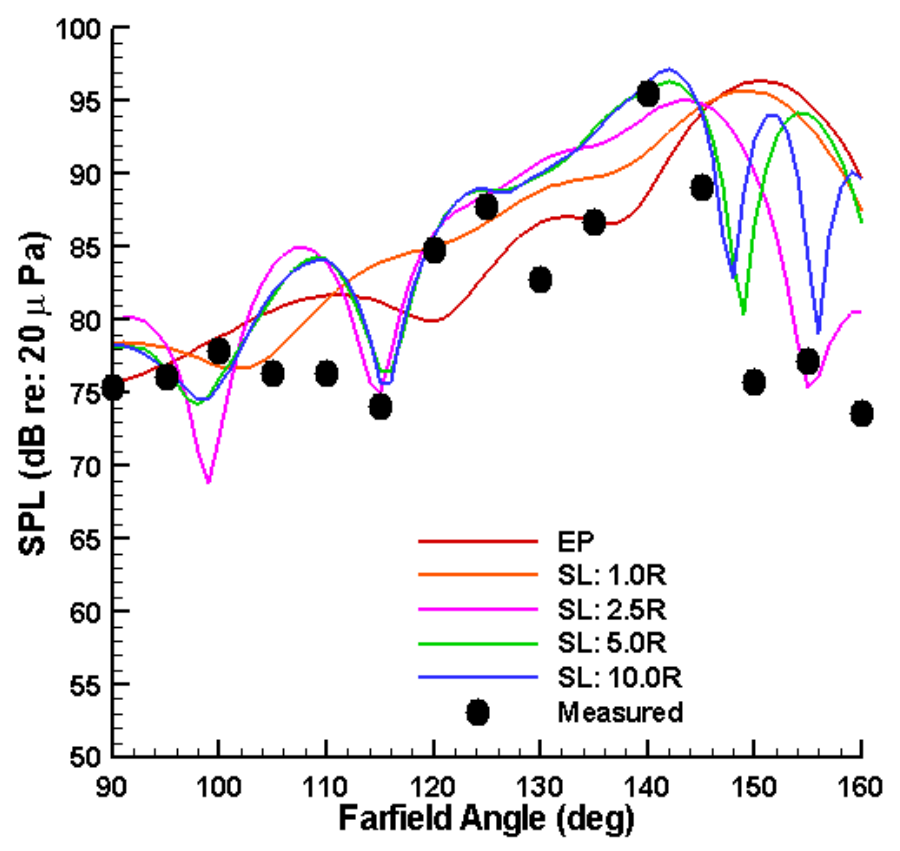

Figure 19. Aft ANCF prediction: $2000 \mathrm{RPM}, 3 \mathrm{BPF}(1600 \mathrm{~Hz})$

\section{6 of 19}




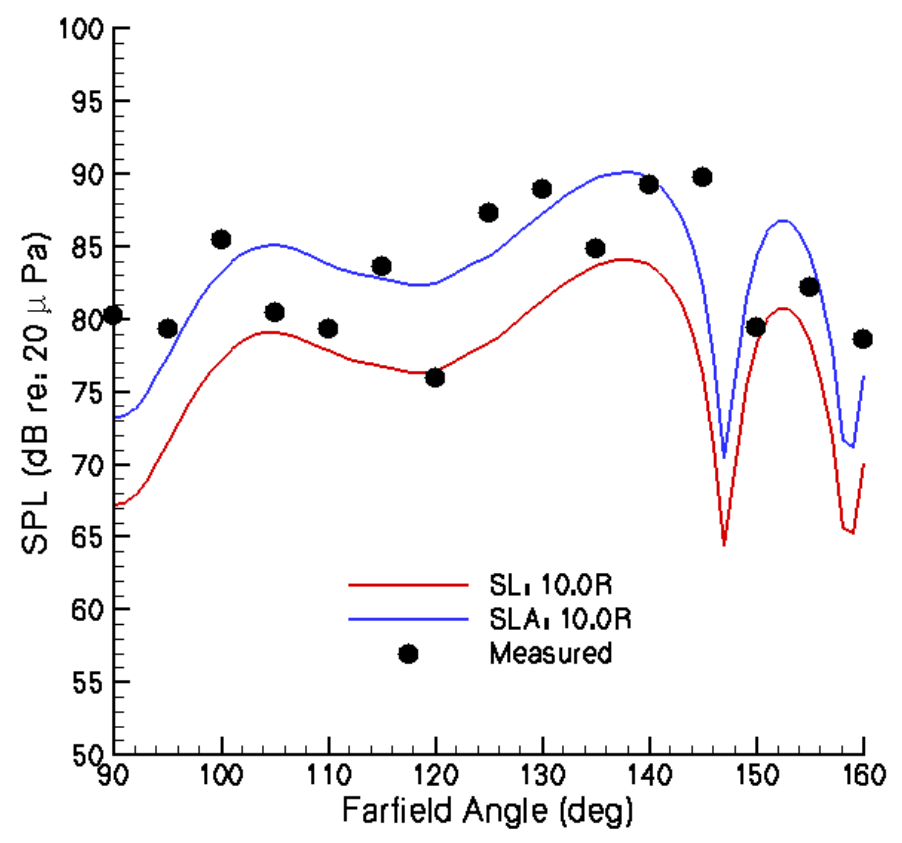

Figure 20. Aft ANCF predictions: $(-10,1)$ mode addition $[2 \mathrm{BPF}=1066 \mathrm{~Hz}]$

\section{Tables}

\begin{tabular}{|c|c|c|}
\hline Mode & Amplitude $\left[\mathrm{N} / \mathrm{m}^{2}\right]$ & Phase [rad] \\
\hline \hline$(2,1)$ & 5.12 & 3.11 \\
\hline$(2,2)$ & 0.56 & 1.74 \\
\hline \hline$(4,1)$ & 4.53 & 2.41 \\
\hline$(4,2)$ & 1.07 & -2.67 \\
\hline$(4,3)$ & 0.47 & -1.01 \\
\hline \hline$(6,1)$ & 1.27 & 1.68 \\
\hline$(6,2)$ & 0.70 & 1.58 \\
\hline$(6,3)$ & 0.30 & 1.46 \\
\hline$(6,4)$ & 0.28 & 1.45 \\
\hline$(-8,1)$ & 1.68 & 1.73 \\
\hline$(-8,2)$ & 0.83 & -0.75 \\
\hline$(-8,3)$ & 0.18 & -2.37 \\
\hline
\end{tabular}

Table 1. Source Information for 1700 RPM $\left(M_{d}=0.144\right)$ 


\begin{tabular}{|c|c|c|}
\hline Mode & Amplitude $\left[\mathrm{N} / \mathrm{m}^{2}\right]$ & Phase [rad] \\
\hline \hline$(2,1)$ & 5.16 & 2.08 \\
\hline$(2,2)$ & 0.91 & 0.61 \\
\hline \hline$(4,1)$ & 6.16 & -0.92 \\
\hline$(4,2)$ & 2.38 & 0.15 \\
\hline$(4,3)$ & 0.46 & 2.35 \\
\hline \hline$(6,1)$ & 7.40 & 0.86 \\
\hline$(6,2)$ & 0.47 & -0.27 \\
\hline$(6,3)$ & 1.51 & 0.92 \\
\hline$(6,4)$ & 0.42 & -2.34 \\
\hline$(-8,1)$ & 3.13 & 1.47 \\
\hline$(-8,2)$ & 0.62 & -3.04 \\
\hline$(-8,3)$ & 0.12 & -1.32 \\
\hline
\end{tabular}

Table 2. Source Information for 1800 RPM $\left(M_{d}=0.154\right)$

\begin{tabular}{|c|c|c|}
\hline Mode & Amplitude $\left[\mathrm{N} / \mathrm{m}^{2}\right]$ & Phase [deg] \\
\hline \hline$(2,1)$ & 7.82 & 2.14 \\
\hline$(2,2)$ & 1.44 & 1.70 \\
\hline \hline$(4,1)$ & 2.59 & -0.62 \\
\hline$(4,2)$ & 1.27 & 1.75 \\
\hline$(4,3)$ & 1.67 & 2.45 \\
\hline \hline$(6,1)$ & 7.70 & -0.64 \\
\hline$(6,2)$ & 0.93 & -2.81 \\
\hline$(6,3)$ & 0.96 & 3.04 \\
\hline$(6,4)$ & 0.45 & 1.80 \\
\hline$(-8,1)$ & 2.11 & 0.44 \\
\hline$(-8,2)$ & 1.19 & -2.53 \\
\hline$(-8,3)$ & 1.33 & 0.34 \\
\hline
\end{tabular}

Table 3. Source Information for $1900 \mathrm{RPM}\left(M_{d}=0.164\right)$

18 of 19 


\begin{tabular}{|c|c|c|}
\hline Mode & Amplitude [N/m $\left.{ }^{2}\right]$ & Phase [deg] \\
\hline \hline$(2,1)$ & 8.14 & 0.04 \\
\hline$(2,2)$ & 2.01 & -0.80 \\
\hline \hline$(4,1)$ & 1.91 & 2.73 \\
\hline$(4,2)$ & 1.19 & 2.07 \\
\hline$(4,3)$ & 0.28 & 0.67 \\
\hline \hline$(6,1)$ & 1.97 & -0.99 \\
\hline$(6,2)$ & 1.48 & 2.10 \\
\hline$(6,3)$ & 0.49 & 0.88 \\
\hline$(6,4)$ & 0.45 & 1.81 \\
\hline$(-8,1)$ & 3.29 & -1.08 \\
\hline$(-8,2)$ & 2.13 & 0.92 \\
\hline$(-8,3)$ & 2.27 & -0.89 \\
\hline$(-8,4)$ & 0.43 & -0.72 \\
\hline
\end{tabular}

Table 4. Source Information for 2000 RPM $\left(M_{d}=0.175\right)$

\begin{tabular}{|c|c|c|c|}
\hline Case & Pseudo-Duct Length & Axial Grid Dimension & Total Wall-Clock Time [s] \\
\hline \hline EP & 0 & 0 & 3 \\
\hline SL: $1.0 \mathrm{R}$ & $\mathrm{R}$ & 33 & 13 \\
\hline SL: $2.5 \mathrm{R}$ & $2.5 \mathrm{R}$ & 81 & 27 \\
\hline SL: $5.0 \mathrm{R}$ & $5.0 \mathrm{R}$ & 161 & 51 \\
\hline SL: $10.0 \mathrm{R}$ & $10.0 \mathrm{R}$ & 321 & 103 \\
\hline
\end{tabular}

Table 5. Grid Sizes and Complete Calculation Timings for Various 'Pseudo-Ducts'

19 of 19 